\title{
ON METRIC PROPERTIES OF SETS OF ANGULAR LIMITS OF MEROMORPHIC FUNCTIONS
}

\author{
J. E. MCMILLAN*
}

Let $f$ be a nonconstant function meromorphic in the unit disc $D=\{|z|<1\}$, with circumference $C$, and let $E_{z}$ be a subset of $C$ with positive (linear) measure. Suppose that at each $\zeta \in E_{z}, f$ has an angular limit $a_{\zeta}$, and let $E_{w}=\left\{a_{\zeta}: \zeta \in E_{z}\right\}$. It is known that $E_{w}$ contains a closed set with positive harmonic measure (see Priwalow [6, p. 210] or Tsuji [7, p. 339]). Also known is that even when $f$ is a schlicht function mapping $D$ onto the interior of a Jordan curve, it may happen that $E_{w}$ has linear measure zero (see Lavrentieff [2]); and a recent theorem of Matsumoto [4, p. 133] states, in effect, that if $f$ is a schlicht function mapping $D$ onto the interior of a Jordan curve, then $E_{w}$ cannot have $\frac{1}{2}$-dimensional measure zero (For the definitions of (exterior) linear measure and $\alpha$-dimensional measure zero $(\alpha>0)$, see $[5$, pp. 149, 150].). The purpose of the present paper is to prove a theorem that generalizes Matsumoto's theorem. As a corollary of our theorem, we obtain: If each point of $E_{w}$ is accessible (with a Jordan arc) through the complement of $f(D)=\{f(z): z \in D\}$, then $E_{w}$ contains a closed set that does not have $\frac{1}{2}$-dimensional measure zero.

If $E_{w}$ is all of the extended $w$-plane $\Omega$, the desired conclusion already holds ; so that we may, by first subjecting $\Omega$ to a linear transformation, assume that $\infty \notin E_{w}$. Our result is most conveniently expressed in terms of the Riemann surface $S$ of $f$ over $\Omega$. For each $\zeta \in E_{z}$ and positive number $h$, let $S(\zeta, h)$ be the component of $S$ over $\left\{\left|w-a_{\zeta}\right|<h\right\}$ such that if $r$ is sufficiently near $1(r$ $<1)$, then $r \zeta$ corresponds under $f$ to a point of $S(\zeta, h)$; and let $P S(\zeta, h)$ be the projection of $S(\zeta, h)$ onto $\Omega$.

We prove

Theorem. Suppose that to each $\zeta \in E_{z}$ there correspond a Jordan arc $r_{\zeta}$ (contained in the finite $w$-plane) with one endpoint $a_{\zeta}$ and a positive number $h_{\zeta}$ such

Received July 7, 1955.

* I wish to thank Professor Bagemihl for his help. 
that $P S\left(\zeta, h_{\zeta}\right) \cap r_{\zeta}=\phi . \quad$ Then $E_{w}$ contains a closed set that does not have $\frac{1}{2}$-dimensional measure zero.

Proof. Let $m(E)$ and $m_{e}(E)$ denote the (linear) measure and exterior (linear) measure of the set $E \subset C$. From Lusin's theorem, there exists a closed set $E_{z}^{(1)} \subset E_{z}$ such that $m\left(E_{z}^{(1)}\right)>0$ and

(1) the restriction of $a_{\zeta}$ to $E_{z}^{(1)}$ is a continuous function.

For each $\zeta \in E_{z}^{(1)}$, let $\Delta_{\zeta}$ be an open (Euclidean) disc with rational radius and center with two rational coordinates such that

$$
a_{\zeta} \in A_{\zeta} \subset\left\{\left|w-a_{\zeta}\right|<h_{\zeta}\right\},
$$

and let $S_{\zeta}$ be the component of $S$ over $\Delta_{\zeta}$ such that if $r$ is sufficiently near 1 $(r<1)$, then $r \zeta$ corresponds under $f$ to a point of $S_{\xi}$. Then $P S_{\zeta} \cap \gamma_{\zeta}=\phi$. Since there are only countably many distinct $S_{\zeta}$, there exists $\zeta_{0} \in E_{z}^{(1)}$ such that the set

$$
E_{z}^{(2)}=\left\{\zeta \in E_{z}^{(1)}: S_{\zeta}=S_{\zeta_{0}}\right\}
$$

has positive exterior measure. Let $S_{0}=S_{\zeta_{0}}$ and $\Delta_{0}=\Delta_{\zeta_{0}}$. Then

(2) for each $\zeta \in E_{z}^{(2)}, P S_{0} \cap \gamma_{\zeta}=\phi$ and $a_{\zeta} \in \Delta_{0}$.

Let $S(\zeta, r)$ denote the sector $\left(\zeta=e^{i \tau}, 0<r<1\right)$

$$
\left\{\zeta+\rho e^{i \theta}: 0<\rho<r, \tau+\frac{3 \pi}{4}<\theta<\tau+\frac{5 \pi}{4}\right\},
$$

and for each $\zeta \in E_{z}^{(2)}$, let $r_{\zeta}$ be a positive number such that

(3) $f\left(S\left(\zeta, r_{\zeta}\right)\right) \subset \Delta_{0}$.

Let $r$ be a positive number and $E_{z}^{(3)}$ a subset of $E_{z}^{(2)}$ such that $m_{e}\left(E_{z}^{(3)}\right)>0$, and for each $\zeta \in E_{z}^{(3)}, r \leq r_{\zeta}$. Let $r^{\prime}\left(0<r^{\prime}<1\right)$ be such that $\left\{|z|=r^{\prime}\right\}$ intersects the rectilinear segments on the boundary of $S(1, r)$, and let $I$ be a component of $\left\{r^{\prime}<|z|<1\right\} \cap \cup S(\zeta, r)$, the union being taken over all $\zeta \in E_{z}^{(3)}$, such that the set

$$
E_{z}^{(4)}=\left\{\zeta \in E_{z}^{(3)}: S(\zeta, r) \cap I \neq \phi\right\}
$$

has positive exterior measure. Then

$$
I=\left\{r^{\prime}<|z|<1\right\} \cap \cup S(\zeta, r),
$$


where the union is taken over all $\zeta \in \bar{E}_{z}^{(4)}$ (the bar denotes closure). Thus $I$ is the interior of a rectifiable Jordan curve $\Gamma$, and

$$
\bar{E}_{z}^{(4)}=\Gamma \cap C \subset E_{z}^{(1)}
$$

From (3) we have $f(I) \subset \Delta_{0}$, and it follows that $I$ corresponds under $f$ to a subset of $S_{0}$. Thus $f(I) \subset P S_{0}$, and from (2) we have

(4) for each $\zeta \in E_{z}^{(4)}, f(I) \cap \gamma_{\zeta}=\phi$.

Let $l$ be a positive constant, and let $E_{z}^{(5)}$ be a subset of $E_{z}^{(4)}$ such that $m_{e}\left(E_{z}^{(5)}\right)>0$ and

(5) for each $\zeta \in E_{z}^{(5)}$, the diameter of $\gamma_{\zeta}$ is greater than or equal to $2 l$.

By making suitable linear transformations, we may suppose that

$$
0 \in I \text { and } f(0)=\infty \text {. }
$$

Let $\gamma$ be an arbitrary Jordan arc joining $(0<r<l, a \in \Omega-\{\infty\})\{|w-a|=$ $r\}$ to $\{|w-a|=l\}$ and lying, except for its endpoints, in $\{r<|w-a|<l\rangle$. Let $\omega(w ; a, r, \gamma)$ denote the harmonic measure of $\{|w-a|=r\}$ with respect to $\Omega-[\{|w-a| \leq r\} \cup \gamma]$. Using Matsumoto's argument [4, pp. 134, 135], we now prove that there exist positive constants $h$ and $M$ (which are independent of $a, r$ and $\gamma$ ) such that

$$
\omega(\infty ; a, r, r) \leq M \sqrt{r} \quad(0<r<h) .
$$

By letting $\gamma^{\prime}$ denote the image of $r$ under the translation $w-a$ and noting that

$$
\omega(\infty ; a, r, \gamma)=\omega\left(\infty ; 0, r, \gamma^{\prime}\right),
$$

we see that we need only prove (7) under the assumption that $a=0$. We assume then that $a=0$, and write

$$
D_{r}=\{|w|<r\}, \quad C_{r}=\{|w|=r\} .
$$

Let $\omega_{r}(w)$ be the harmonic measure of $C_{r}$ with respect to

$$
\Omega-\left[\bar{D}_{r} \cup\{u+i v: r \leq u \leq l, v=0\}\right] .
$$

Then from Matsumoto's Lemma 2 [4, p. 132], there exist positive constants $h$ and $M$ such that $(h<l)$

$$
\omega_{r}(\infty) \leq M \sqrt{r} \quad(0<r<h) .
$$


Now let $r$ be a fixed number satisfying $0<r<h$. For each $r^{\prime}$ satisfying $r<r^{\prime}$ $<l$, let $\gamma_{r}$, be the subarc of $r$ that joins $C_{r}$, to $C_{l}$ and lies, except for its endpoints, in $\left\langle r^{\prime}<|w|<l\right\}$. And let $\left\{J_{n}\right\}$ be a sequence of Jordan curves such that $\bar{D}_{r}$ is contained in the exterior of $J_{n}, J_{n+1}$ is contained in the interior $I_{n}$ of $J_{n}$, and $\gamma_{r^{\prime}}=\bigcap_{n=1}^{\infty} I_{n}$. Then the harmonic measure $\omega_{n}(w)$ of $C_{r}$ with respect to $\Omega-\left[\bar{D}_{r} \cup J_{n} \cup I_{n}\right]$ and the harmonic measure $\omega^{\prime}(w)$ of $C_{r}$ with respect to $\Omega-\left[\bar{D}_{r} \cup r_{r}\right]$ satisfy

$$
\omega_{n}(\infty) \uparrow \omega^{\prime}(\infty) .
$$

For a fixed $n$, we choose rectilinear segments

$$
L_{j}=\left\{w: r_{j} \leq|w| \leq r_{j+1}, \text { argument } w=\theta_{j}\right\}
$$

$\left(j=1, \ldots, k ; r^{\prime}=r_{1}<r_{2}<\cdots<r_{k+1}=l\right)$ that are contained in $I_{n}$. Then the harmonic measure $\omega_{n}^{*}(w)$ of $C_{r}$ with respect to $\Omega-\left[\bar{D}_{r} \cup \bigcup_{j=1}^{k} L_{j}\right]$ satisfies the relation $\omega_{n}(\infty) \leq \omega_{n}^{*}(\infty)$; and from Matsumoto's Lemma 1 [4, p. 131], the harmonic measure $\widetilde{\omega}(w)$ of $C_{r}$ with respect to

$$
\Omega-\left[\bar{D}_{r} \cup\left\{u+i v: r^{\prime} \leq u \leq l, v=0\right\}\right]
$$

satisfies the relation $\omega_{n}^{*}(\infty) \leq \widetilde{\omega}(\infty)$. Thus $\omega_{n}(\infty) \leq \widetilde{\omega}(\infty)$, and from (9) we have the relation $\omega^{\prime}(\infty) \leq \widetilde{\omega}(\infty)$; and letting $r^{\prime} \downarrow r$, we see that $\omega(\infty ; 0, r, r)$ $\leq \omega_{r}(\infty)$. Thus from (8) the proof of $(7)$ is complete.

We now suppose that the set $E_{w}^{(1)}=\left\{a_{\zeta}: \zeta \in E_{z}^{(1)}\right\}$ (which is closed and bounded because $E_{z}^{(1)}$ is closed, $\infty \notin E_{w}$, and (1)) has $\frac{1}{2}$-dimensional measure zero. We wish to prove that this assumption leads to a contradiction. Let $E_{w}^{*}=\bar{E}_{w}^{(5)}$, where $E_{w}^{(5)}=\left\{a_{\zeta}: \zeta \in E_{z}^{(5)}\right\}$. Then $E_{w}^{*} \subset E_{w}^{(1)}$. Let $E_{z}^{*}=\left\{\zeta \in E_{z}^{(1)}\right.$ : $\left.a_{\zeta} \in E_{w}^{*}\right\}$. Then from (1), $E_{z}^{*}$ is closed relative to the closed set $E_{z}^{(1)}$, and is therefore closed.

Let $\varepsilon$ be a positive number. Since $E_{w}^{*}$ is closed and bounded and has $\frac{1}{2}$-dimensional measure zero, there exists a finite number of discs $\Delta_{j}=\left\{\left|w-a_{j}\right|\right.$ $\left\langle r_{j}\right\}(j=1, \ldots, n)$ such that

$$
\begin{gathered}
0<r_{j}<h \quad(j=1, \ldots, n), \\
\sum_{j=1}^{n} \sqrt{r_{j}}<\frac{\varepsilon}{M} . \\
E_{w}^{*} \subset \bigcup_{j=1}^{n} \Delta_{j},
\end{gathered}
$$


and

$$
\Delta_{j} \cap E_{w}^{*} \neq \phi \quad(j=1, \ldots, n) .
$$

It follows from (13) that for each $j(j=1, \ldots, n)$ there exists $\zeta_{j} \in E_{z}^{(5)}$ such that $a_{\zeta j} \subseteq \Delta_{j}$; and from (5) we see that $\gamma_{\zeta j} \cap\left\langle\left|w-a_{j}\right|=l\right\} \neq \phi$. Thus we may let $\gamma_{j}$ be a subarc of $\gamma_{\zeta j}$ that joins $\left\{\left|w-a_{j}\right|=r_{j}\right\}$ to $\left\{\left|w-a_{j}\right|=l\right\}$ and lies, except for its endpoints, in $\left\{r_{j}<\left|w-a_{j}\right|<l\right\}$. Let $U$ be the component of $\Omega-\bigcup_{j=1}^{n}\left[\bar{\Delta}_{j} \cup \gamma_{j}\right]$ that contains $\infty$, and let

$$
\omega(w)=\sum_{j=1}^{n} \omega\left(w ; a_{j}, r_{j}, r_{j}\right) \quad(w \in U) .
$$

Then from (7), (10) and (11), we have

$$
\omega(\infty)<\varepsilon .
$$

Let $z\left(z^{\prime}\right)$ be a conformal mapping of $D^{\prime}=\left\{\left|z^{\prime}\right|<1\right\}$ onto $I$ such that $z(0)=$ 0 (recall $(6))$. Since $E_{z}^{*}$ is closed and $E_{z}^{(5)} \subset E_{z}^{*}, m\left(E_{z}^{*}\right)>0$; and it follows that $E_{z}^{*}$ corresponds under $z=z\left(z^{\prime}\right)$ to a closed set $E_{z^{\prime}}^{*}$ on $C^{\prime}=\left\{\left|z^{\prime}\right|=1\right\}$; and since $I$ is rectifiable, $m\left(E_{z^{\prime}}^{*}\right)>0\left[6\right.$, p. 127]. Let $u\left(z^{\prime}\right)$ be the harmonic measure of $E_{z^{\prime}}^{*}$ with respect to $D^{\prime}$. Let $F\left(z^{\prime}\right)=f\left(z\left(z^{\prime}\right)\right)$, let $D_{0}$ be the component of $\left\{z^{\prime} \in D^{\prime}: F\left(z^{\prime}\right) \in U\right\}$ that contains 0 (recall (6)), and let $B$ denote the boundary of $D_{0}$.

We wish now to establish the relation

$$
u\left(z^{\prime}\right) \leq \omega\left(F\left(z^{\prime}\right)\right) \quad\left(z^{\prime} \in D_{0}\right) .
$$

From (4) we see that

$$
F\left(B \cap D^{\prime}\right) \subset \bigcup_{j=1}^{n}\left\{\left|w-a_{j}\right|=r_{j}\right\}-\bigcup_{j=1}^{n} r_{j},
$$

so that in particular,

$$
\lim _{z^{\prime} \rightarrow \zeta, z^{\prime} \in \nu_{0}} \omega\left(F\left(z^{\prime}\right)\right) \geq 1 \quad \text { for each } \zeta \in B \cap D^{\prime} .
$$

It follows from (4) and a theorem of MacLane [3, p. 10] that for each $j(j=$ $1, \ldots, n)$, the level set $\left\{z^{\prime} \in D^{\prime}:\left|F\left(z^{\prime}\right)-a_{j}\right|=r_{j}\right\}$ "ends at points of $C^{\prime}$ " [3, p. 8]. Thus it follows from (16) that each point of $B \cap C^{\prime}$ ' is accessible through $D_{0}$ (that is, for each $\zeta \in B \cap C^{\prime}$ there exists a Jordan arc that is, except for the one endpoint $\zeta$, contained in $D_{0}$ ). Since at each point of $E_{z^{\prime}}^{*}, F$ has an asymptotic value that is in $E_{w}^{*}$, we have from (12) that each point of $E_{z^{\prime}}^{*}$ is 
accessible through $D^{\prime}-D_{0}$. Thus, each point of $E_{z^{\prime}}^{*} \cap B$ is accessible through both $D_{0}$ and $D^{\prime}-D_{0}$, and from a theorem of Bagemihl [1, Theorem 1], the set $E_{z^{\prime}}^{*} \cap B$ is countable. But for each $\zeta \in C^{\prime}-E_{z^{\prime}}^{*}, \lim _{z^{\prime} \rightarrow \zeta} u\left(z^{\prime}\right)=0$, so that (15) follows from an extension of the maximum principle.

From (15) and (14) we have

$$
\frac{1}{2} m\left(E_{z^{\prime}}^{*}\right)=u(0) \leq \omega(F(0))=\omega(\infty)<\varepsilon,
$$

and since $\varepsilon$ is arbitrary, we have a contradiction; and the proof of the theorem is complete.

Remark. Let $E=E\left(p_{0} p_{1} \cdots\right)$, where $p_{n}=n$, be the Cantor-type set defined by Nevanlinna $[5$, p. 154]. Then $E$ has positive harmonic measure $[5$, p. 155] and for each positive number $\alpha$, since $2^{n} /(n !)^{\alpha} \rightarrow 0(n \rightarrow \infty), E$ has $\alpha$-dimensional measure zero. Let $F$ be a holomorphic function that maps $D$ one-to-one and conformally onto the universal covering surface of $\Omega-[E \cup\{\infty\}]$. It follows from theorems of Nevanlinna [5, pp. 208, 213] that $F$ has angular limits at almost all (except for a set of measure zero) points of $C$; and from a theorem of Lusin and Priwalow [6, p. 212], at almost every point of $C$ the angular limit value of $F$ is in $E$. Applying now an argument of Lusin and Priwalow (see $[6$, p. 210]) we see that there exists a nonconstant function $f$ bounded and analytic in $D$ such that for each positive number $\alpha, E_{w}$ has $\alpha$-dimensional measure zero.

\section{REFERENCES}

[1] F. Bagemihl: Curvilinear cluster sets of arbitrary functions, Proc. Nat. Acad. Sci. U.S.A., 41 (1955), 379-382.

[2] M. Lavrentieff: Sur quelques problèmes concernant les fonctions univalentes sur la frontière, Rec. Math., 43 (1936), 816-846 (en russe).

[3] G. R. MacLane: Asymptotic values of holomorphic functions, Rice Univ. Studies, 49 (1963), 1-83.

[4] K. Matsumoto: On some boundary problems in the theory of conformal mappings of Jordan domains, Nagoya Math. Journ., 24 (1964), 129-141.

[5] R. Nevanlinna: Eindeutige analytische Funktionen, Berlin-Göttingen-Heidelberg. 1953.

[6] I. I. Priwalow: Randeigenschaften analytischer Funktionen, Berlin, 1956.

[7] M. Tsuji: Potential theory in modern function theory, Tokyo, 1959.

\section{University of Wisconsin-Milwaukee}

